\title{
Keck all sky precision adaptive optics: project overview
}

Wizinowich, P., Chin, J., Correia, C., Lu, J., Brown, T., et al.

P. Wizinowich, J. Chin, C. Correia, J. Lu, T. Brown, K. Casey, S. Cetre, J.-R. Delorme, L. Gers, L. Hunter, S. Lilley, S. Ragland, A. Surendran, E. Wetherell, A. Ghez, T. Do, T. Jones, M. Liu, D. Mawet, C. Max, M. Morris, T. Treu, S. Wright, "Keck all sky precision adaptive optics: project overview," Proc. SPIE 11448, Adaptive Optics Systems VII, 114480E (13 December 2020); doi: 10.1117/12.2560017

SPIE. Event: SPIE Astronomical Telescopes + Instrumentation, 2020, Online Only 


\title{
Keck All Sky Precision Adaptive Optics
}

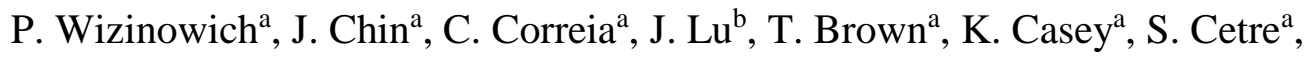
J-R. Delorme ${ }^{\mathrm{a}}$, L. Gers ${ }^{\mathrm{a}}$, L. Hunter ${ }^{\mathrm{c}}$, S. Lilley ${ }^{\mathrm{a}}$, S. Ragland ${ }^{\mathrm{a}}$, A. Surendran ${ }^{\mathrm{a}}$, E. Wetherell ${ }^{\mathrm{a}}$,

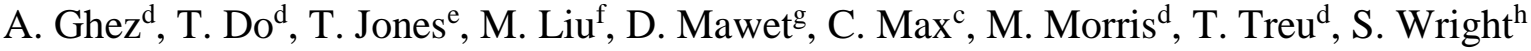

\author{
${ }^{\mathrm{a}}$ W. M. Keck Observatory \\ ${ }^{\mathrm{b}}$ University of California Berkeley \\ 'University of California Santa Cruz \\ dUniversity of California Los Angeles \\ eUniversity of California Davis \\ ${ }^{\mathrm{f}}$ University of Hawaii \\ ${ }^{\mathrm{g}}$ Caltech

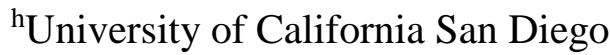

\begin{abstract}
We present the status and plans for the Keck All sky Precision Adaptive optics (KAPA) program. KAPA includes four key science programs, an upgrade to the Keck I laser guide star (LGS) adaptive optics (AO) facility to improve image quality and sky coverage, AO telemetry based point spread function (PSF) estimates for all science exposures, and an educational component focused on broadening the participation of women and underrepresented groups in instrumentation. For the purpose of this conference we will focus on the AO facility upgrade which includes implementation of a new laser, wavefront sensor and real-time controller to support laser tomography, the laser tomography system itself, and modifications to an existing near-infrared tip-tilt sensor to support multiple natural guide star (NGS) and focus measurements.
\end{abstract}

Keywords: adaptive optics, laser tomography, near-infrared sensing, PSF reconstruction

\section{INTRODUCTION}

Thanks to funding from the NSF Mid-Scale Innovation Program the W.M. Keck Observatory (WMKO) embarked on a major new five-year AO initiative as of September 2018. KAPA consists of four key science programs, an upgrade to the Keck I AO facility ${ }^{1}$ and an education program.

The key science programs are focused on the following challenges:

1. Constraining dark matter, the Hubble constant, and dark energy via strong gravitational lensing

2. Testing General Relativity and studying supermassive black hole interactions at the Galactic Center

3. Characterizing galaxy kinematics and metallicity using rare highly magnified galaxies

4. Directly studying gas-giant protoplanets around the youngest stars

Additional funding to support these science programs, and the associated science tool development, has been provided by the Gordon and Betty Moore Foundation.

The KAPA education program has elements ranging from undergraduate to post-graduate level with an overall goal of broadening participation in instrumentation for women and underrepresented minorities. Funding for the associated AstroTech summer instrumentation workshop has been provided by the Heising-Simons Foundation.

The goal of the KAPA upgrade is to improve the image quality delivered to OSIRIS ${ }^{2}$ with greater sky coverage, and to improve the quantitative science results by providing PSF estimates with each science exposure. OSIRIS is a nearinfrared integral field spectrograph and imager. The elements of the KAPA upgrade are illustrated in Figure 1.

A TOPTICA/MPBC laser, identical to the laser previously implemented on Keck II, ${ }^{3}$ has been implemented to replace the Keck I LMCT laser. This was done to increase the sodium return (by a factor of 10 to allow multiple laser guide stars) and to improve the laser reliability. The new laser has been in science operation since May 2020.

Adaptive Optics Systems VII, edited by Laura Schreiber, Dirk Schmidt, Elise Vernet, Proc. of SPIE

Vol. 11448, 114480E · C 2020 SPIE · CCC code: 0277-786X/20/\$21 - doi: 10.1117/12.2560017 


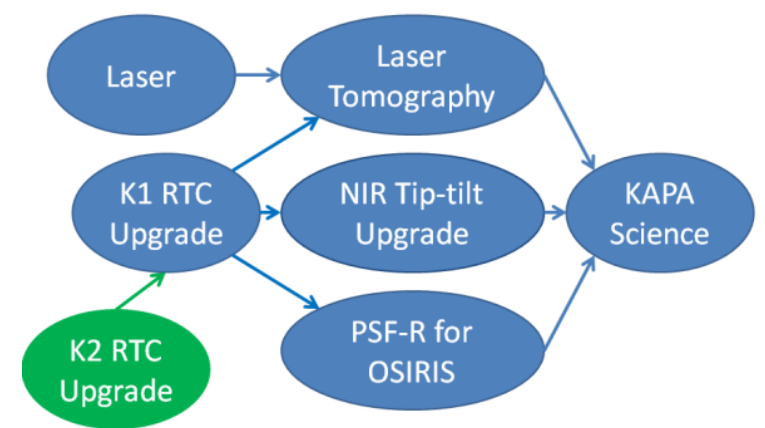

Figure 1: Technical elements of the KAPA upgrade to the Keck I AO facility

We are building on the separately NSF MRI funded Keck II real-time controller (RTC) upgrade (which completed its detailed design review in July 2019). The RTC upgrade includes a new larger format, lower noise wavefront sensor camera (OCAM2K). The KAPA project will implement an identical system on Keck I; the interfaces and algorithms needed to support multiple LGS and NGS will be added. The implementation will occur first on Keck I for KAPA.

Multiple LGS will be used to reduce the "cone" effect. The laser tomography upgrade includes four components: a modification to the laser beam transport system to produce four LGS (the asterism generator), a modification to the wavefront sensor camera optics to sense all four LGS on the same detector, RTC modifications to support four LGS and a laser tomography algorithm, and an LGS asterism simulator system to support daytime calibration and testing.

Multiple NGS are needed to determine the tip-tilt in the direction of the science object. This will be implemented with the existing Keck I near-infrared tip-tilt sensor. ${ }^{4}$ We will also be extending our NIRC2 PSF-reconstruction (PSF-R) approach to multiple LGS and OSIRIS.

The physical location of the KAPA components with the Keck I AO system is shown schematically in Figure 2.

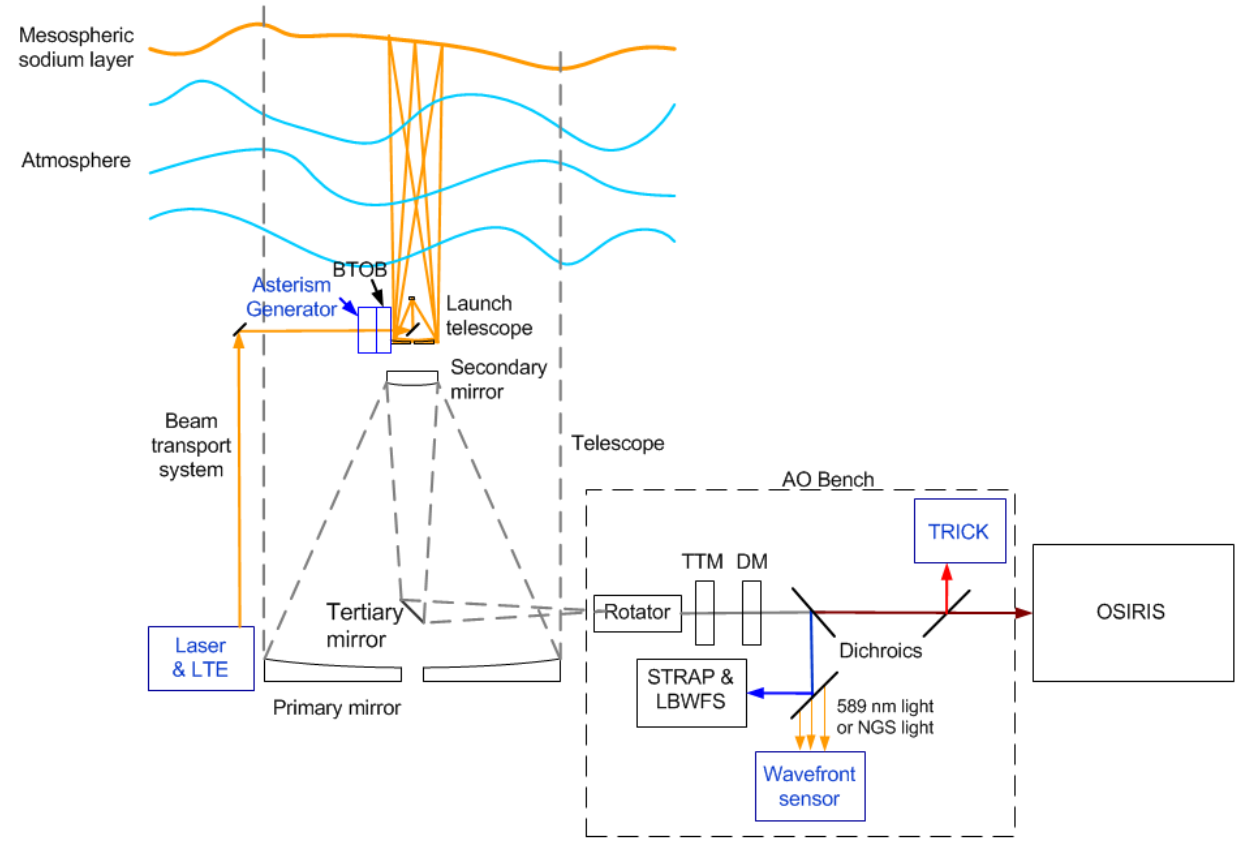

Figure 2: Physical location of KAPA related hardware (identified in blue text). The new laser head and laser table enclosure (LTE) are mounted on the telescope elevation ring. The LTE provides laser formatting and disagnostics prior to the beam entering the beam transport system. The laser electronics and supporting control systems are located on the right Nasmyth platform. The asterism generator is mounted in the secondary module just before the beam transport optics bench (BTOB). The near-infrared tip-tilt sensor (TRICK) and new wavefront sensor camera are mounted on the $\mathrm{AO}$ bench. 
The following sections follow the sequence shown in Figure 1. These sections are followed by overviews of the predicted performance, science tools, science products and the education program.

\section{LASER GUIDE STAR FACILITY}

As part of KAPA, the previous LMCT solid-state laser has been replaced with a TOPTICA/MPBC Raman-fiber amplifier laser for higher sodium return, higher reliability and lower maintenance. The new laser facility, including some beam train modifications, has been in science operation since May 2020. The on-sky laser performance is quite similar to that for the Keck II laser ${ }^{3}$.
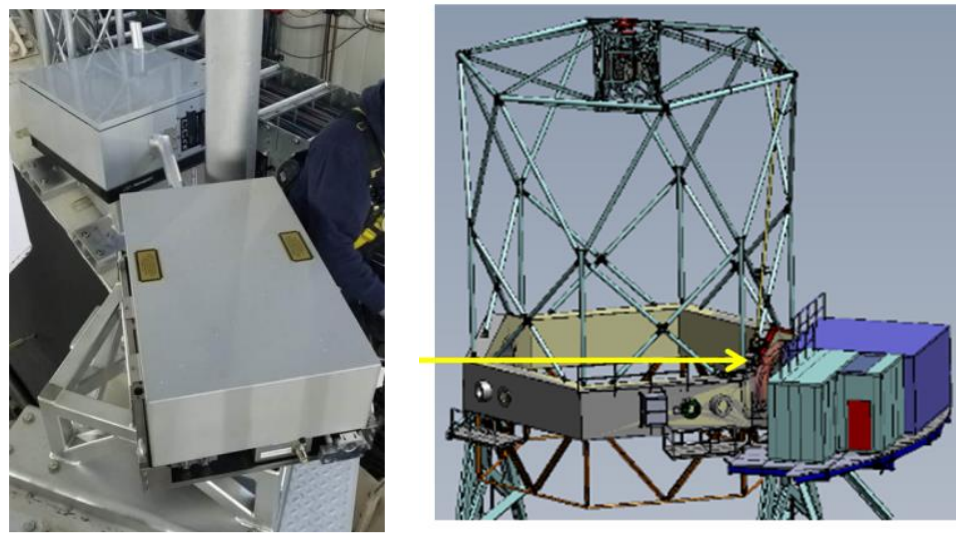

Figure 3. Left: Laser head (bottom white box) and laser table enclosure (upper gray box) mounted on the telescope elevation ring. The output of the laser table enclosure connects to the existing laser beam train. The Nasmyth platform is on the right. Right: Telescope with existing laser room (light blue) on the right Nasmyth platform. The yellow arrow points to the location of the laser head on the elevation ring.

\section{REAL-TIME CONTROLLER}

The RTC is being developed by a consortium led by Microgate, including Swinburne University of Technology, Australian National University, and Observatoire de Paris, with expertise in the areas of hardware systems and controls, GPU computing, AO and simulations. Members were also part of the European Green Flash Consortium, working toward a common computational architecture for the ELT. The RTC factory acceptance is scheduled for January 2021.

The RTC architecture is shown in Figure 4. Microgate (consortium) is responsible for delivering the interface module (IM), computational engine (CE), and telemetry recorder server (TRS). The Keck I and II AO systems have eleven science modes requiring the RTC to be capable of interfacing with the Shack-Hartmann sensor (using the current SciMeasure camera or new OCAM2K camera), the near-infrared pyramid wavefront sensor (PWS), the near-infrared tip-tilt sensor (TRICK), the STRAP tip-tilt sensor, the Xinetics deformable mirror, and the downlink (DTT) and uplink (UTT) tip-tilt mirrors. It will also interface with a future MEMS DMs on Keck II and three additional UTT mirrors on Keck I. To reduce the footprint and impact of the RTC, only the IM will be located near the AO optics bench. A fiber link capable of $40 \mathrm{GbE}$ transfers both the sensor data to the $\mathrm{CE}$ and command data from the CE. This allows the $\mathrm{CE}$ and the TRS to be co-located in a temperature controlled computer room.

The CE is a Linux Server based on the CACAO (Compute and Control for AO) architecture of low latency, shared memories, and semaphores. The CE is based on NVidia's TESLA V100 TESLA boards with the CUDA development environment. Currently, two VT100 TESLA boards are planned for the CE; one for the real-time computer and one to support functionalities such as PSF-R, sensor fusion and predictive control. The second VT100 also provides redundancy in case of GPU failure. Raw and processed data are stored onto a 100TB TRS with 12 nights of capacity in the most data intensive mode.

Within the CE, the software architecture uses modules such as KRAKEN (high level sequencing and user interface) and OCTOPUS (interface to shared memory) to configure the software and pass data directly to/from the GPUs. These modules, along with the layout of the business units (BUs), provide the user flexibility to modify or add algorithms for future needs. The use of semaphores, persistent kernels, shielding and direct memory transfers of data to the GPUs 
provides low latency $(<250 \mu \mathrm{s})$ and jitter performance meeting the KAPA timing requirements. The COMPASS simulator framework is added to support simulation and analysis of the various modes.

The existing SciMeasure wavefront sensor camera (Figure 5 (left)) will be replaced with an OCAM2K camera (right). The reducer optics that relay the Shack-Hartmann lenslet images to the detector with the right scale (i.e. going from $200 \mu \mathrm{m}$ lenslet spacing to 4 pixel spacing) will be replaced with new optics allowing sampling of four LGS. Two OCAM2K cameras have been received and Nutek is scheduled to deliver the reducer optics in November 2020.

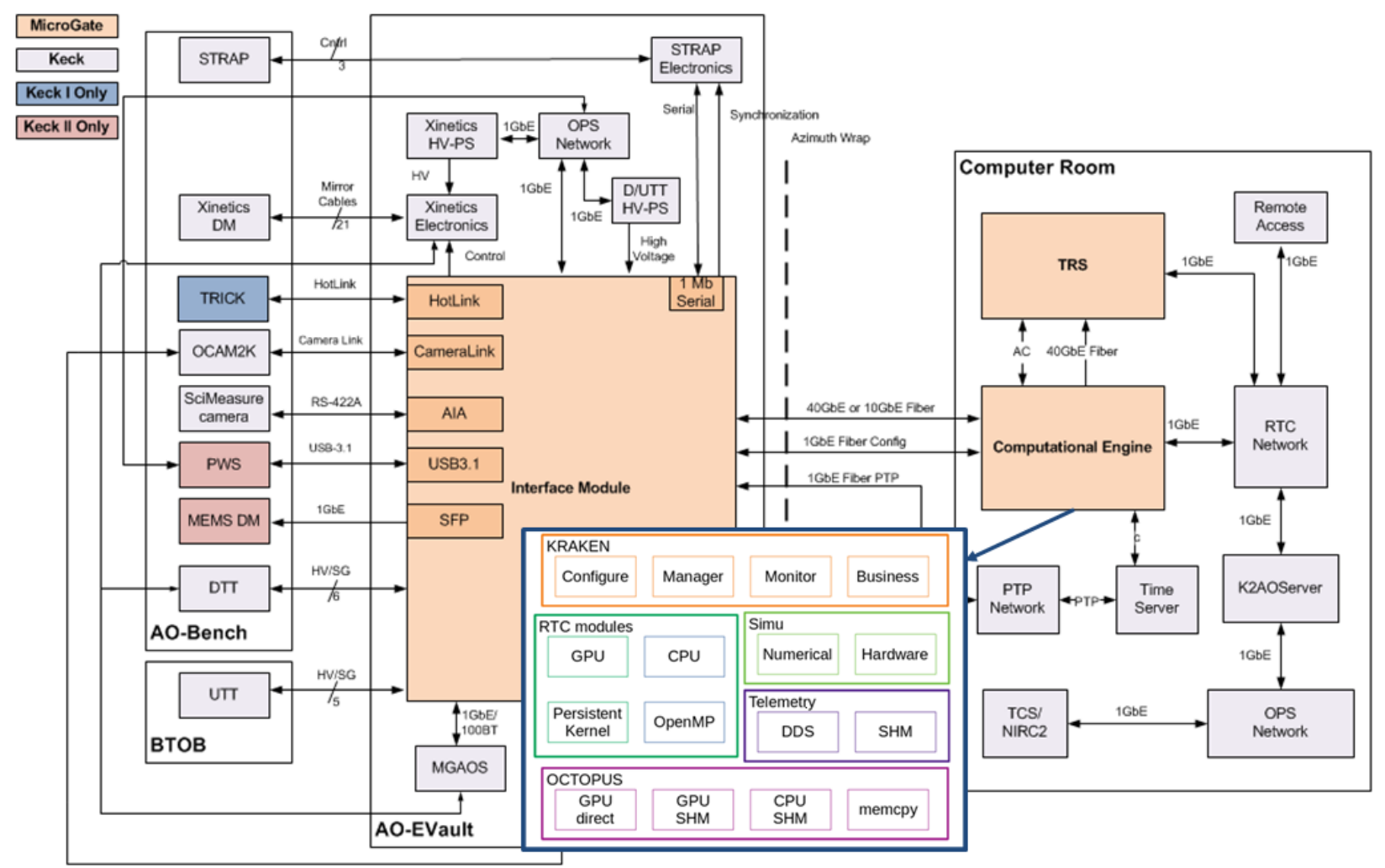

Figure 4: Real-time controller architecture. The inset box at bottom-center shows the components on the Computational Engine.
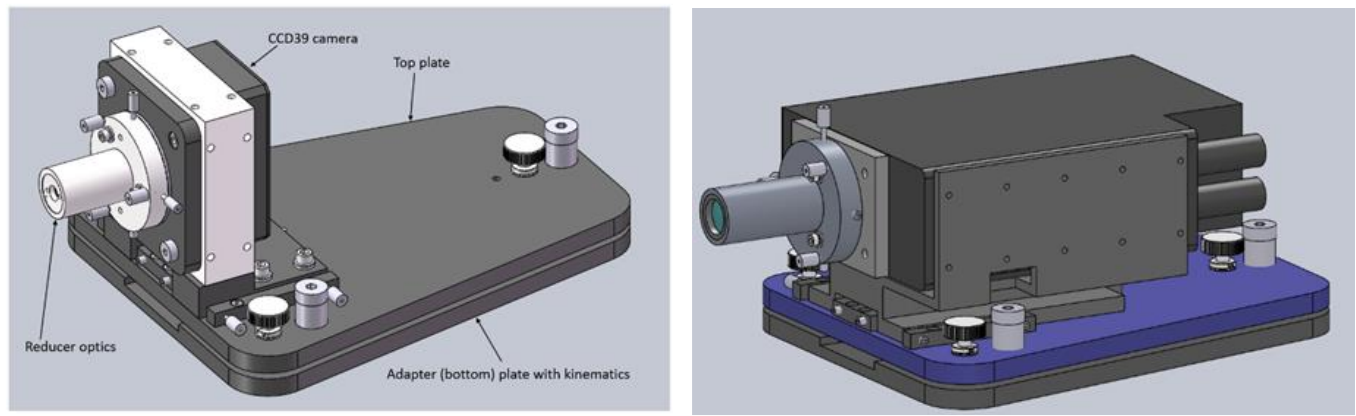

Figure 5: Current SciMeasure (left) and new OCAM2K (right) wavefront sensor camera installation.

\section{LASER TOMOGRAPHY}

\subsection{Asterism Radius and Wavefront Sensor}

Four LGS on an equilateral triangle with a 7.6" radius will be used for KAPA laser tomography. The number of LGS and radius were selected based on a combination of simulations and what radius would fit on the OCAM2K wavefront sensor camera. The predicted high order wavefront error is shown in Figure 6. The location of the four LGS pupils on the wavefront sensor CCD is shown in Figure 7. The wavefront sensor optics will include four field stops on the 
appropriate radius followed by four sets of pupil relay optics to reimage the telescope pupil on the lenslet array. A schematic of the wavefront sensor opto-mechanics and the pupil relay is provided in Figure 8.

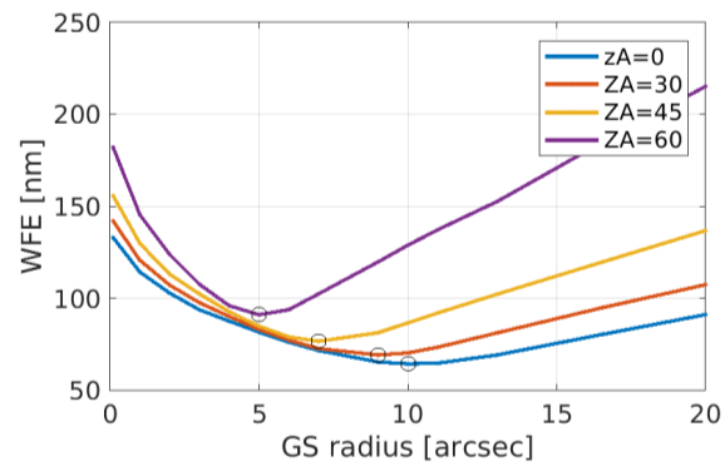

Figure 6: High-order wavefront error (tip-tilt removed) for median seeing conditions as a function of the guide star (GS) asterism radius (4 LGSs on a square) and ZA. The minima are indicated with black circles - almost perfect evolution with 1/airmass.

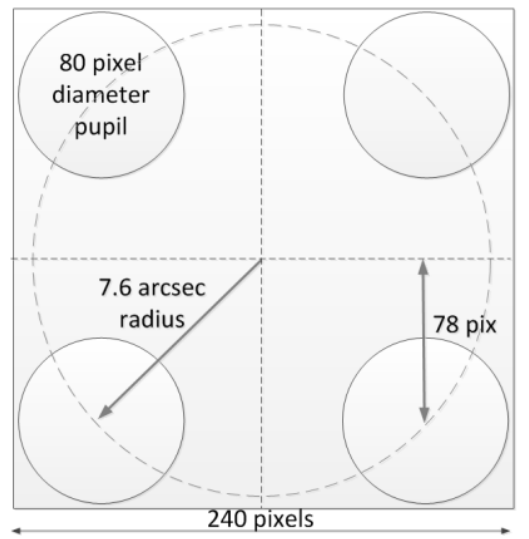

Figure 7: The location of the four LGS pupils on the on the OCAM2K detector.
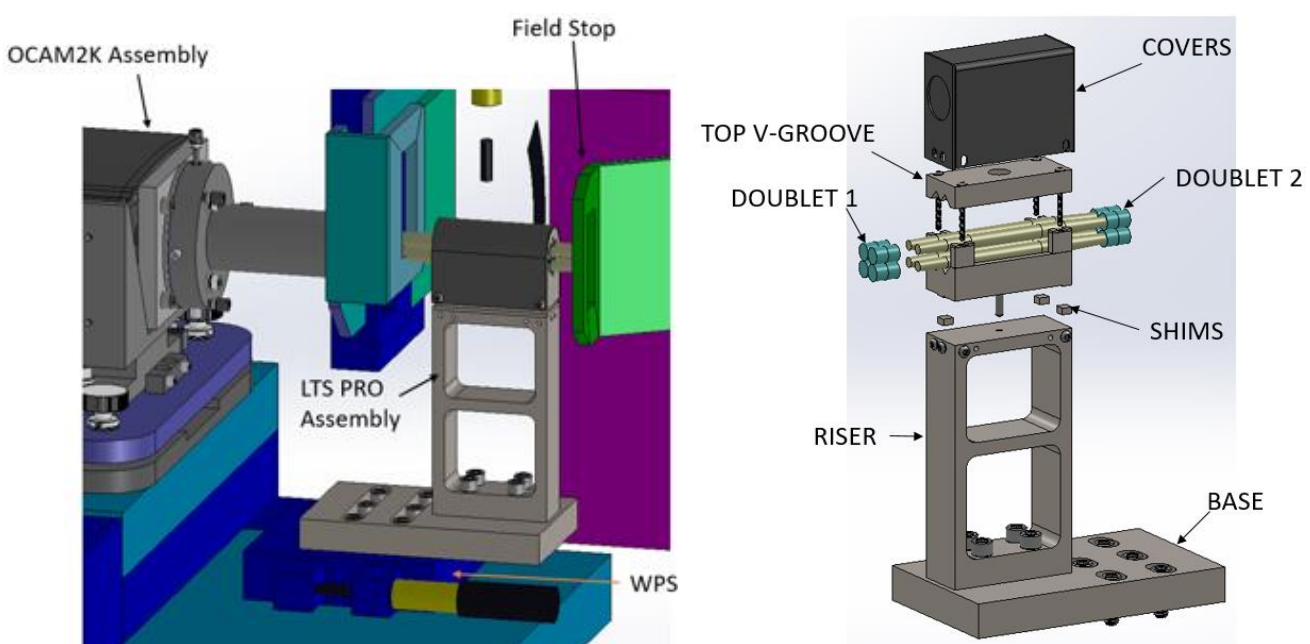

Figure 8: Left: Wavefront sensor schematic showing (from right to left) the field stop, pupil relay optics (PRO), lenslet array, reducer optics mounted to the OCAM2K camera and the OCAM2K camera. Right: Exploded view of the pupil relay containing a pair of doublets for each of the four LGS. 


\subsection{Asterism Generator}

The asterism generator is required to produce the four LGS asterism from a single input laser beam. This system will be located in the secondary module of the telescope shown in Figure 9, 2 $\mathrm{m}$ before the laser launch telescope. The generator, shown in Figure 10 and Figure 11, is mounted on a rotation stage to keep the LGS asterism fixed with respect to the LGS wavefront sensor, and on a translation stage to allow removal for single LGS operation. Three nonpolarizing beamsplitter cubes and a prism are used to send the resultant four beams to fast tip-tilt mirrors. The tip-tilt mirrors are mounted at the appropriate angle to produce the required asterism radius on the sky. The beam compressor moves the four beams closer together so they will overlap on the launch telescope secondary mirror (the exit pupil). Opto-Mécanique de Précision (OMP) in Canada has been contracted to provide the asterism generator. The asterism generator completed its preliminary design in June 2020.

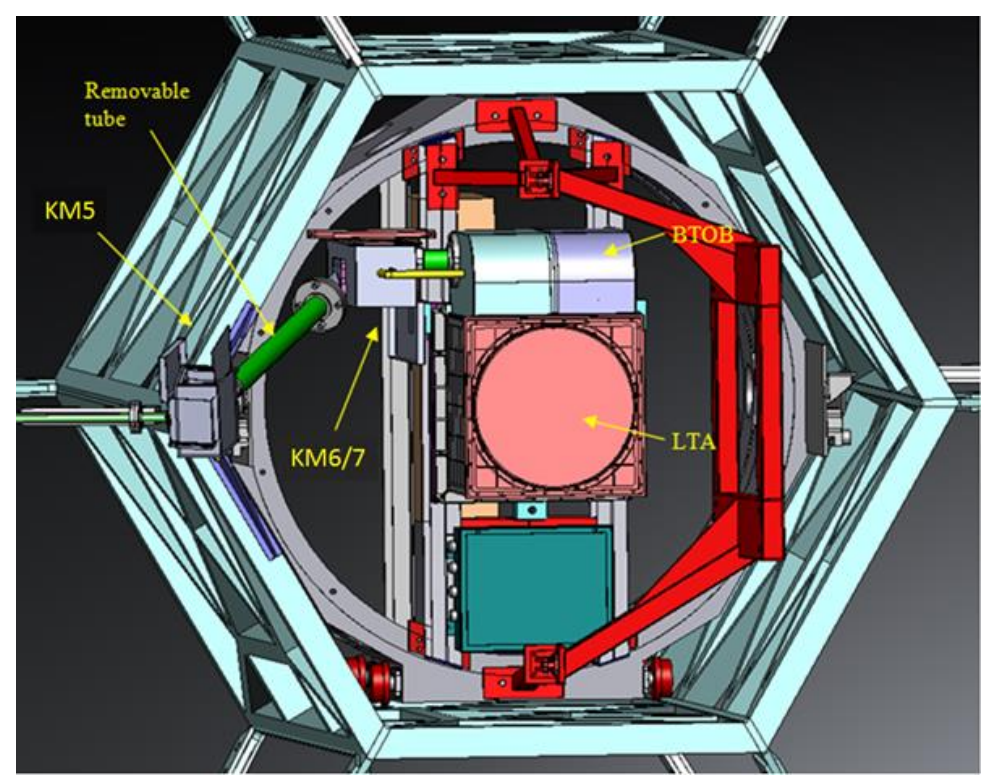

Figure 9: CAD model of the telescope secondary mirror module showing some of the laser beam transport mirrors $\left(\mathrm{KM}^{*}\right)$, the beam transfer optics bench (BTOB) which reformats and steers the input laser beam, and the launch telescope assembly (LTA). The asterism generation will be located in the KM6/7 enclosure.

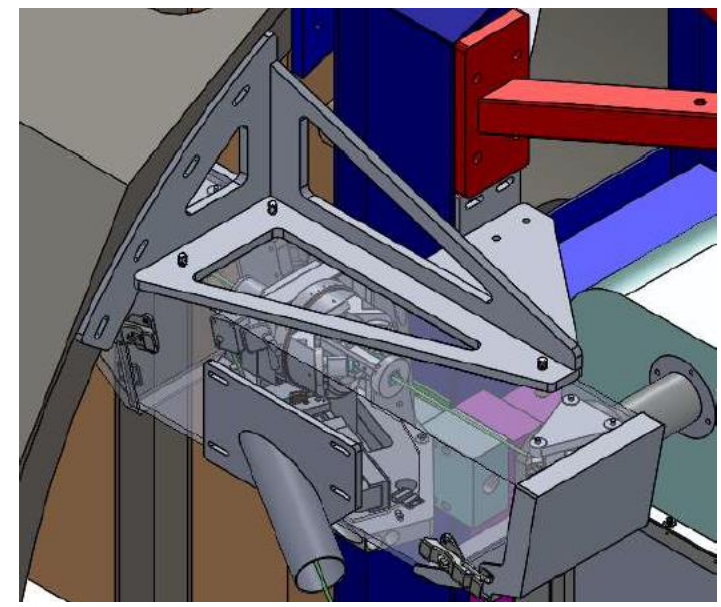

Figure 10: Asterism generator in the KM6/7 enclosure, shown with a transparent view into the enclosure. 

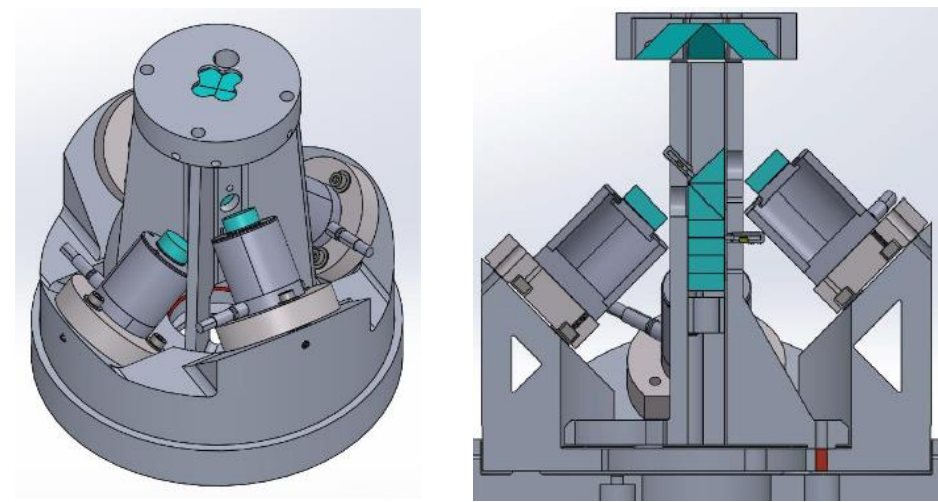

Figure 11: LGS asterism generator. Left: (1) up-link PI piezo actuated mirror mounts; (2) tower body containing the 3 beamsplitter cubes and a right angle prism; (3) tower top containing the beam compressor; and (4) brace system linking these components to the rotator. Right: Cut-away view. The input laser enters from the bottom and the four LGS beams exit at the top.

\subsection{Asterism Simulator and Daytime Testing}

KAPA will include an asterism simulator system to support daytime calibrations and testing. Two fiber bundles (Figure 12) will be procured to simulate the LGS asterism, using $590 \mathrm{~nm}$ light and $\sim 1$ " diameter multi-mode fibers, and a NGS source, using a broad-band source and single-mode fiber diffraction-limited at H and K-band. The output surface of one fiber will be mounted to the fiber "finger" in Figure 13 (left) so that it can be positioned in the AO input focal plane (telescope output focal plane) along with an off-axis single mode (NGS) source. The other bundle will be located at the input focal plane of the telescope simulator shown in Figure 13 (right). The telescope simulator collimates the light through a turbulence phase screen and pupil mask before reconverging the beam with the same f/\# and pupil location as the Keck telescope. The light from the telescope simulator is reflected to the AO input focal plane by a fold mirror on the simulator positioner shown in Figure 13 (left). Figure 14 provides a schematic for the light sources and fiber routing.
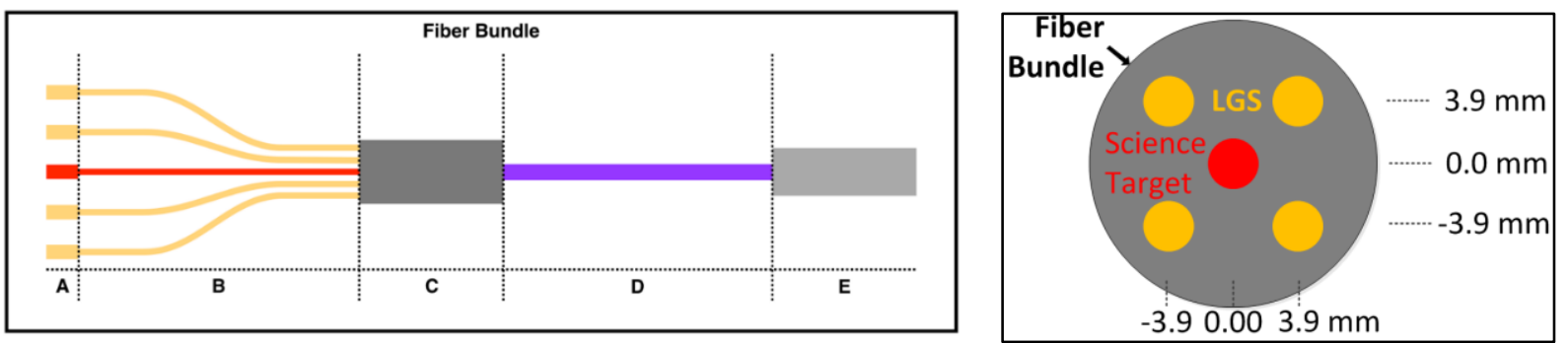

Figure 12: Diagram of the asterism simulator fiber bundle (left) and a face-on view of the output connector. The LGS fibers are multimode on a 7.6" radius and the science target fiber is single mode.

The system-level daytime calibration and testing plan includes the following four phases as the tomography hardware and software are implemented with the AO system:

1. Deployment of the new asterism simulator and its usage for all the daytime tests and calibrations for the NGS and single LGS AO.

2. Deployment of the new telescope/turbulence simulator and its usage for daytime tests and calibrations, with the additional benefit of a telescope pupil. The turbulence altitude, wind speed and r0 should be calibrated during this phase.

3. Integration, calibration and testing of the new pupil relay optics. The first step is to ensure these optics can be used to support NGS and single LGS AO science operations.

4. Laser tomography (LT) AO calibration and testing.

All four phases include closed loop testing, including applying turbulence to the deformable mirror and possibly the tip-tilt mirror. Once Phase 3 is in place turbulence at altitude can be added to the closed loop testing. 

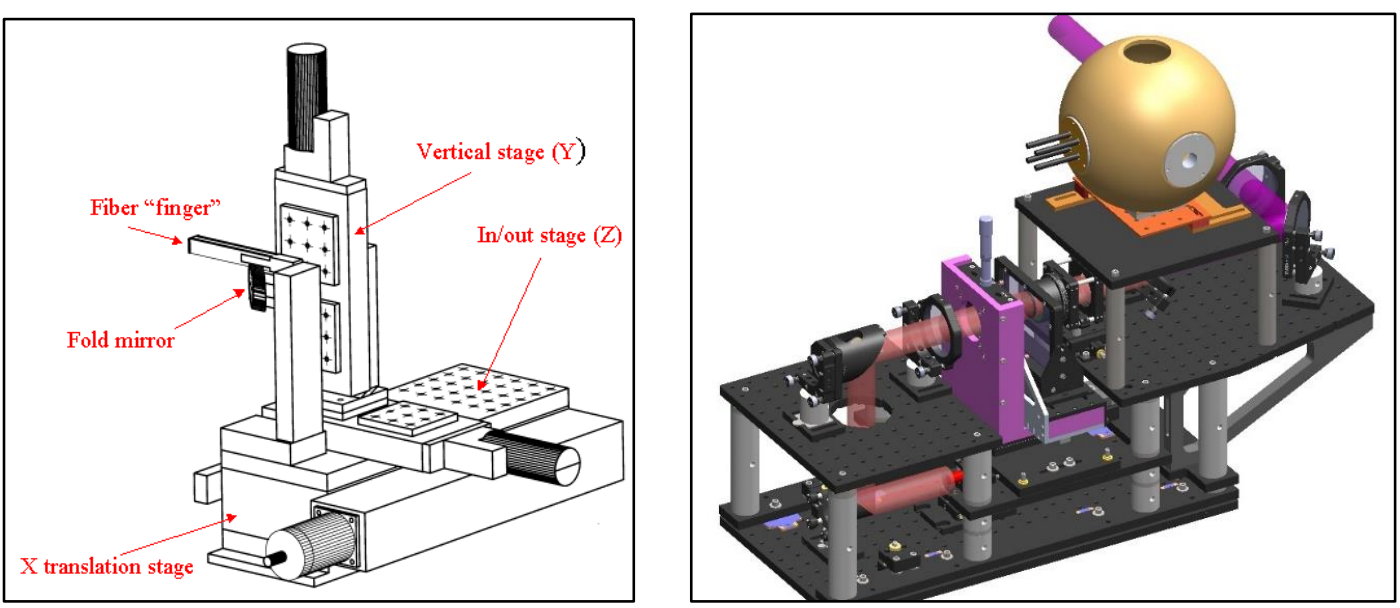

Figure 13: Left: Existing simulator positioner stages which positions a fiber, or a fold mirror to reflect light from a telescope simulator, to produce a source at the AO system's input focal plane. Right: KAPA upgrade to the existing telescope simulator including a phase screen to simulate turbulence at different altitudes.

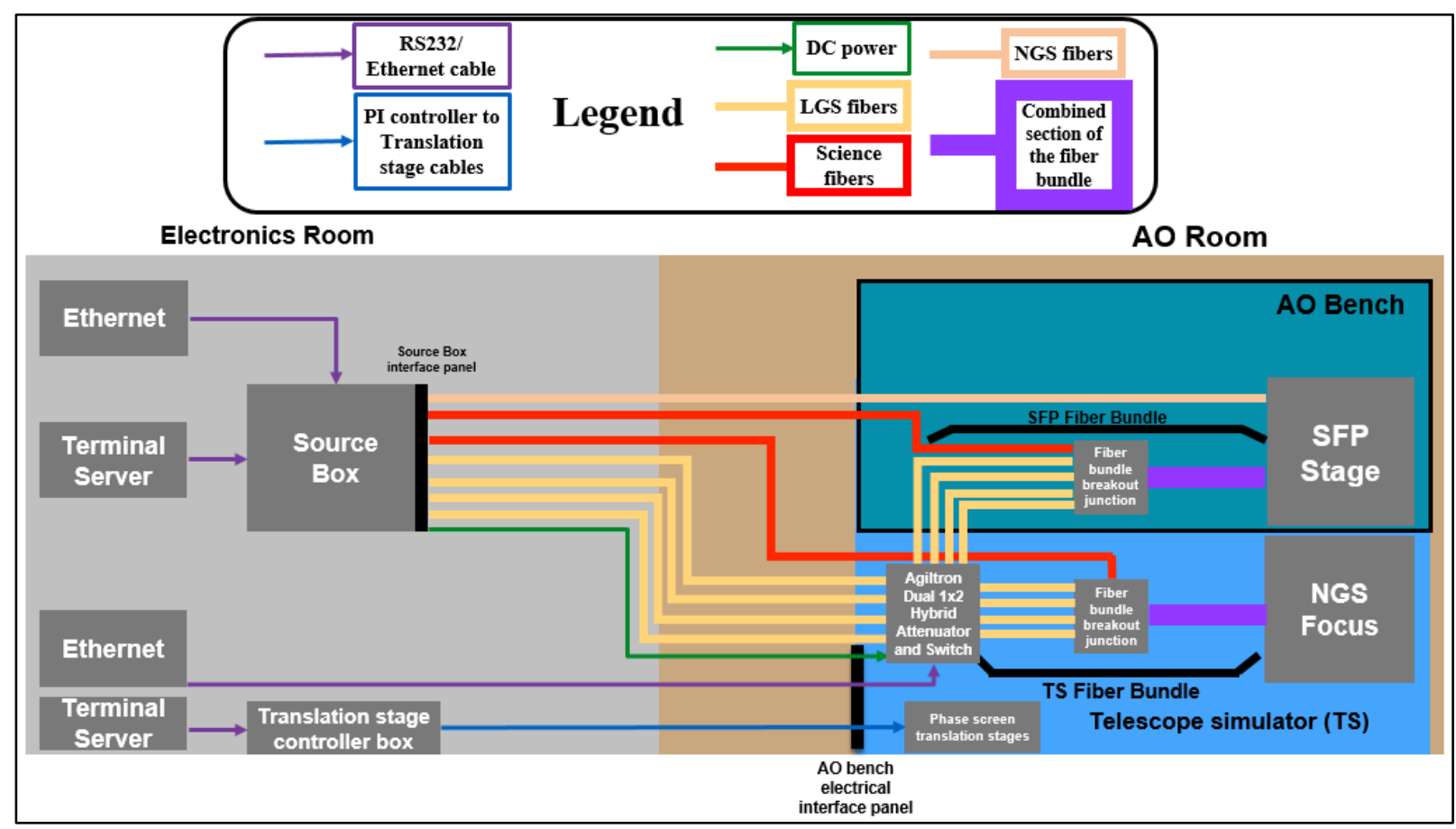

Figure 14: Source simulator schematic. A light source box in the AO electronics room feeds fibers that are mounted on the SFP stage and the telescope simulator.

\subsection{Tomography Algorithm}

The laser tomography algorithms are considerably different from the NGS and single LGS cases in that the advocated regulator is a pseudo open-loop controller (POLC) using a minimum mean-square error (MMSE) tomographic reconstructor. The latter is partially based on a model integrating both geometric system and statistical atmospheric parameters. The POLC consists of two major steps: tomographic reconstruction (spatial) and control (temporal filtering). The focal anisoplanatism error is predicted to be reduced from about $150 \mathrm{~nm}$ rms to the $80 \mathrm{~nm}$ rms level.

The RTC has been designed with considerable extra capacity and for programming flexibility. A prototype GPUbased system is in place at Keck to test the implementation of the hard and soft real-time code in parallel with development and implementation of the KAPA RTC. 


\section{NEAR-INFRARED TIP-TILT SENSOR}

The existing near-infrared tip-tilt sensor is shown in Figure $15 .{ }^{6}$ This system is in shared-risk science usage as of late 2020. An example of the improved performance with this sensor is shown in Figure 16. The KAPA upgrade will include reading out up to three regions of interest centered on the NGS used for tip-tilt sensing to reduce tip-tilt anisoplanatism. Other plans include testing improved methods for system optimization, tip-tilt sensing (e.g. correlation) and sky subtraction.

We are investigating the benefits of using this sensor for slow focus, and possibly astigmatism, measurements using the LIFT algorithm. ${ }^{7}$ Initial on-sky testing have proved encouraging.

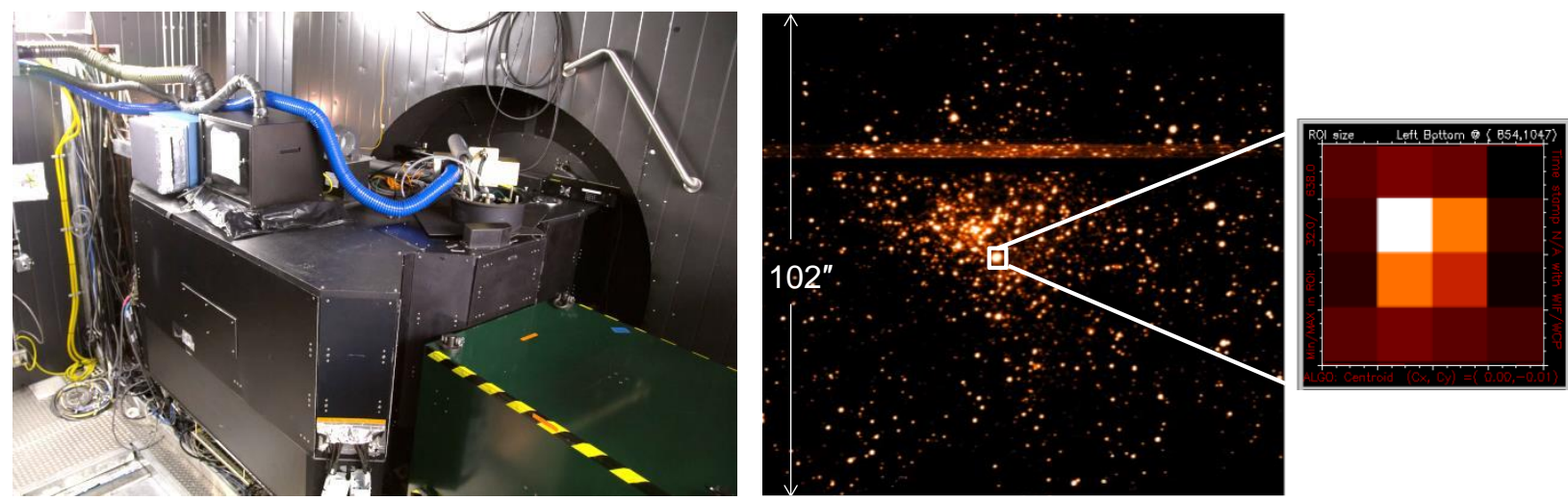

Figure 15: Left: The near-infrared tip-tilt sensor is mounted to the AO bench (blue hose goes to camera electronics) just before the OSIRIS science instrument (green). Center: An image of the Galactic Center taken with the H2RG detector. Right: Multiple reads of one or more regions of interest (a $4 \times 4$ pixel region is shown here) are used to reduce noise.

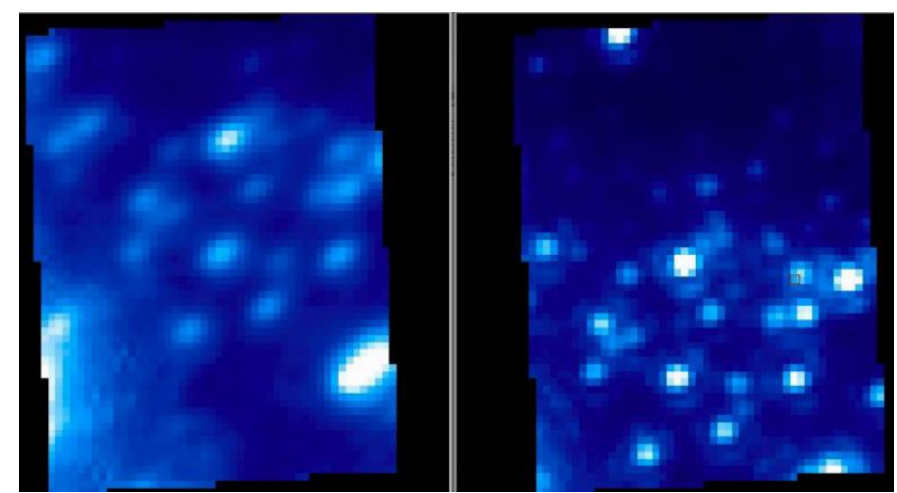

Figure 16: OSIRIS integral field spectrograph images of the Galactic Center. Left using the visible tip-tilt sensor (STRAP) and right using the near-IR tip-tilt sensor (TRICK).

\section{PSF RECONSTRUCTION}

A multi-institutional PSF-R development effort has been undertaken for science with NIRC2 on Keck II. ${ }^{8,9}$ The NIRC2 tool includes an algorithm to reconstruct the on-axis PSF based on the AO telemetry, and algorithms to generate the off-axis components based on the measured instrument off-axis aberrations and atmospheric profiler data. The PSF$\mathrm{R}$ estimates have been successfully demonstrated on-sky for point sources and a science verification phase is currently underway. The nightly data will be processed by pipeline software to provide a grid of PSFs that are archived in the Keck Observatory Archive along with the science data. Some progress has been made to extend these tools for integral field spectroscopy with OSIRIS. ${ }^{10,11}$ The impact of multiple LGS and NGS must be incorporated into both the imaging and integral field spectroscopy PSF-R estimates as part of the KAPA project.

A schematic diagram of the PSF-R facility for NIRC2 science instrument images is shown in Figure 17 with the following elements. 
- A Keck PSF-R block including: (1) The on-axis algorithms used to reconstruct the on-axis PSF from AO telemetry. (2) An off-axis anisoplanatism correction algorithm (an alternative to the baseline UCLA version). (3) A systems science effort and tools to minimize the wavefront errors through improved optimization (e.g. phasing, gain optimization) and calibration of the telescope, AO system and instrument; the goal is to provide a stable on-sky PSF such that high-fidelity end-to-end AO simulations with appropriate system and turbulence parameters should be adequate for PSF-R. (4) The pipeline software, written in IDL, used to generate the PSFs based on the input data.

- A UCLA block including: (1) Instrument characterization of the field dependent aberrations; these aberrations are periodically calibrated using image sharpening and the AO fiber source. (2) The off-axis algorithms. This software tool, called AIROPA, has been installed at Keck. AIROPA includes the use of Maunakea MASS/DIMM data to determine the anisoplanatism correction for PSF-R.

- A LAM block which combines model fitting of focal plane images with AO telemetry based PSF-R. ${ }^{12}$

- PUAKO is a Matlab implementation of the pipeline software blending the Keck and LAM PSF-R algorithm developments (not yet fully tested). This hybrid approach promises to lift some of the challenges of the current approaches (namely the correct estimation of system model parameters).

- Data is archived through the Keck Observatory Archive (KOA) per an interface control document. A grid of reconstructed PSFs will be available with every NIRC2 science exposure to the science community.

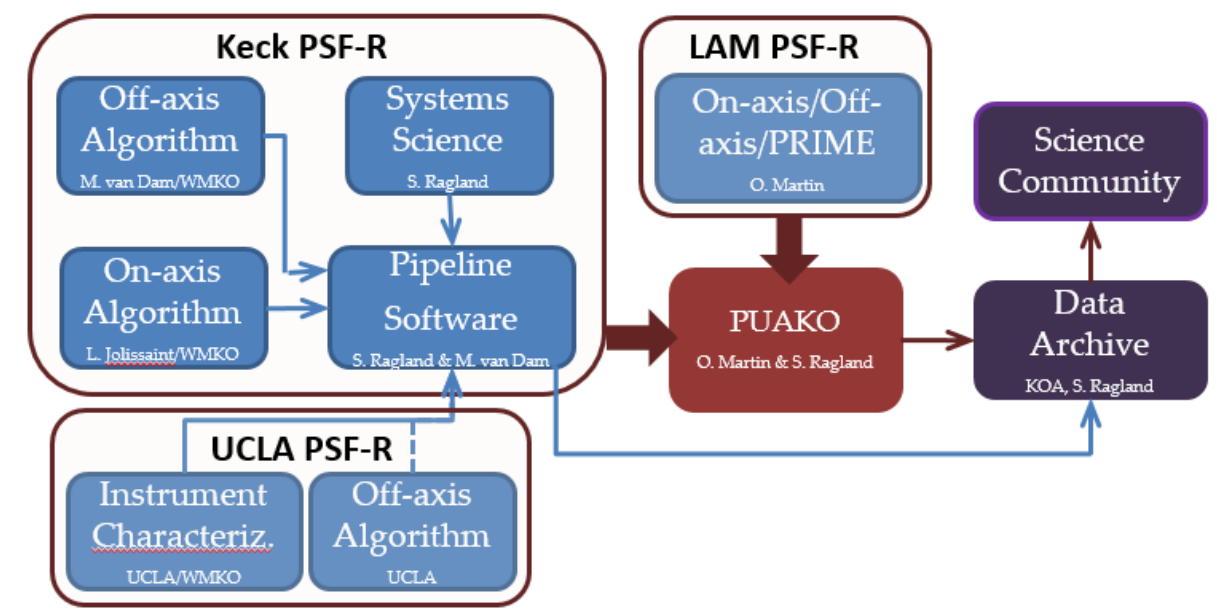

Figure 17: Schematic diagram of the PSF-R facility for NIRC2 AO images.

As part of the KAPA project, the NIRC2 PSF-R tools will be extended to the OSIRIS imager and integral field spectrograph, and the additional OTF terms necessary for laser tomography will be incorporated into the Keck PSF$\mathrm{R}$ module.

\section{PREDICTED PERFORMANCE}

Detailed error budgets and PSF estimates have been developed for KAPA observations of targets representing each of the four key science programs. Two tools have been used to support the performance modeling: a detailed error budget excel spreadsheet and a Matlab-based physical optics simulation model.

The spreadsheet tool was anchored against the current Keck NGS and LGS AO nightly metric data and published Galactic Center data ${ }^{13,14}$. The spreadsheet tool's predicted results match the measured data very well as shown in Table 1. In order to achieve this match an additional high order term of $130 \mathrm{~nm} \mathrm{rms} \mathrm{had} \mathrm{to} \mathrm{be} \mathrm{added} \mathrm{to} \mathrm{all} \mathrm{cases,} \mathrm{and} 6$ mas rms of tip-tilt residual had to be added to the LGS AO cases. The former is believed to be largely due to primary mirror phasing errors that are not seen by the wavefront sensor. 
Table 1: Summary table for the predicted performance in comparison with the measured performance. The last two columns show the high order (HO) and tip-tilt (TT) breakdown for the prediction.

\begin{tabular}{|c|c|c|c|c|c|c|c|}
\hline \multirow{2}{*}{ Case } & \multirow{2}{*}{$\boldsymbol{\lambda}$} & \multicolumn{2}{|c|}{ Strehl Ratio } & \multicolumn{2}{c|}{ FWHM (mas) } & \multicolumn{2}{c|}{ Prediction } \\
\cline { 3 - 6 } & & Meas & Pred & Meas & Pred & HO WFE (nm) & T (mas) \\
\hline Nightly NGS & $\mathrm{K}$ & 0.53 & 0.53 & 50 & 50 & 283 & 4 \\
\hline Nightly LGS & $\mathrm{K}$ & 0.44 & 0.44 & 53 & 53 & 312 & 8 \\
\hline \multirow{2}{*}{ Galactic Center } & $\mathrm{K}$ & 0.30 & 0.30 & 60 & 60 & \multirow{2}{*}{370} & 13 \\
\cline { 2 - 6 } \cline { 3 - 6 } & $\mathrm{H}$ & 0.16 & 0.17 & 64 & 64 & & 13 \\
\hline
\end{tabular}

The Galactic Center current and predicted performance with the RTC and full KAPA upgrades, including using one bright nearby star on TRICK, are shown in Figure 18 under median conditions. KAPA is predicted to achieve a high order wavefront error of $244 \mathrm{~nm} \mathrm{rms}$ with a residual tip-tilt error of 5.6 mas rms on-axis resulting in an H-band Strehl ratio of 0.36 (versus 0.16 currently), and $~ 50 \%$ ensquared energy in a 50 mas integral field spectrograph aperture. Figure 19 provides plots of Strehl ratio and FWHM versus field position from the physical optics simulation model. The resultant PSFs versus field position are provided to the science team for science analysis.

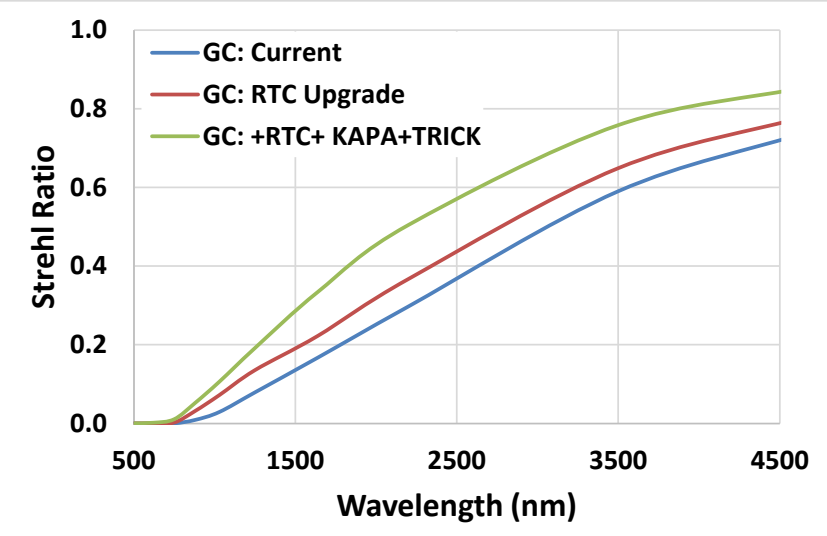

Figure 18: Current (blue) and predicted Galactic Center performance for the RTC (red) and KAPA (green) upgrades.
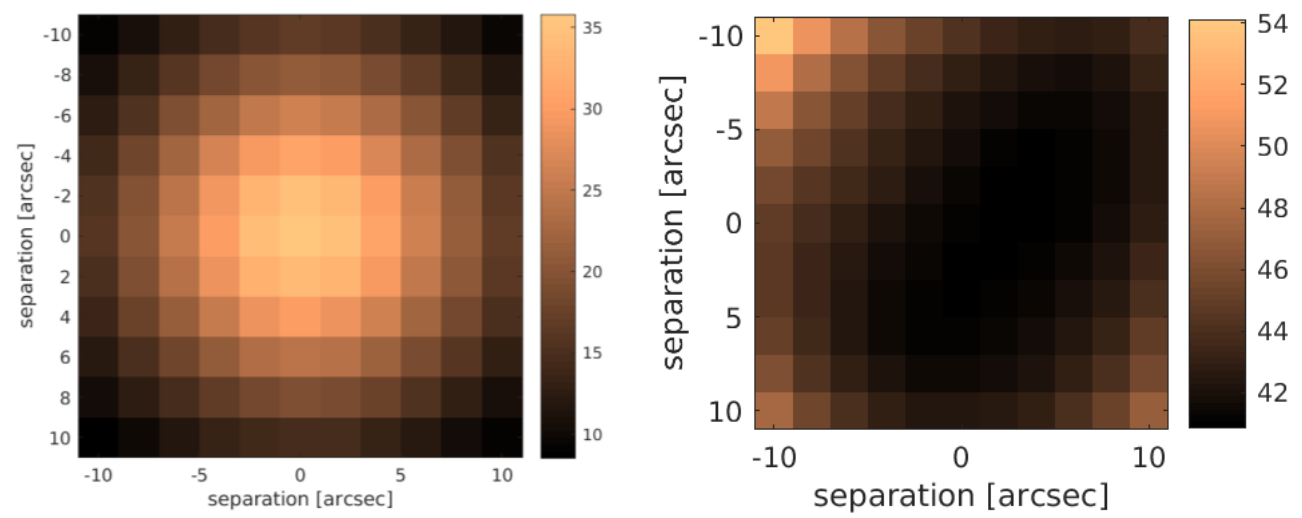

Figure 19: Galactic Center H-band Strehl ratio (left) and FWHM maps for KAPA in median seeing conditions.

For a sample lensed galaxy target the predicted H-band performance is a 0.37 Strehl ratio and 44 mas FWHM, using three near-infrared tip-tilt stars on TRICK, versus the current performance of 0.23 and 53 mas using one visible tiptilt star on STRAP.

KAPA's predicted sky coverage (for galactic latitude $60^{\circ}$, longitude $0^{\circ}$, and $30^{\circ}$ zenith angle) is shown in Figure 20. 


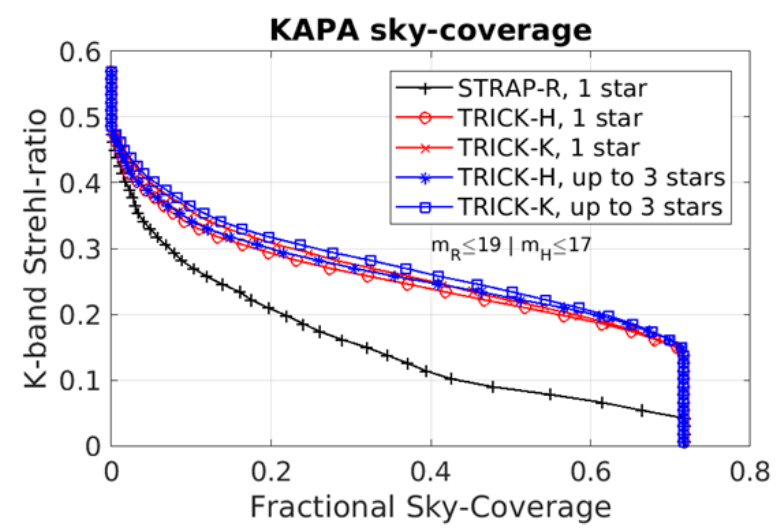

Figure 20: K-band Strehl ratio versus sky coverage estimates for a galactic latitude of $60^{\circ}$, longitude of $0^{\circ}$, and zenith angle of $30^{\circ}$, using STRAP, or TRICK in $\mathrm{H}$ - or K-band.

\section{SCIENCE TOOLS}

Along with the technical and instrumental upgrades that form the primary elements of KAPA, a suite of science tools are being developed. These tools are necessary for observers to plan observations and reduce data, and will be essential in the production of scientific results in each of the four KAPA science surveys. They will also be made broadly available to the Keck community to maximize the total scientific return of KAPA. These tools include:

1. Performance Predictions for survey planning and requirements validation. KAPA PSFs will be provided for each science case. A Strehl ratio and FWHM prediction calculator will be provided for observing planning.

2. OSIRIS Exposure Time Calculator (ETC) for observing planning and science requirements validation. The ETC will use KAPA PSFs and desired observational parameters to predict the SNR.

3. OSIRIS Simulator for the KAPA PSFs through the OSIRIS imager and integral field spectrograph to support experimental design optimization.

4. OSIRIS Data Reduction Pipelines (DRPs) for the imager and improving the existing integral field spectrograph DRP, including additional instrument performance characterization.

5. End-to-end Simulations developed by each science team for their science targets using the KAPA PSFs, including data reduction and science analysis, to determine the quantitative performance and to identify strategies to mitigate residual uncertainties.

6. System Health Monitoring procedures and/or pipelines to regularly monitor basic instrument calibrations, and long term analysis for improved science team data extraction.

\section{KAPA SCIENCE}

Beginning in semester 2023B, subsequent to commissioning of the upgraded system, each science program will perform 20 to 42 nights of science observations spread over three to five years. A number of legacy science products will be released and point spread function (PSF) estimates will be provided for each science exposure in the Keck Observatory Archive (KOA).

To ensure that the four KAPA science programs provide the community with a valuable scientific legacy, the data sets (see Table 2) will be publicly released through the Keck Observatory Archive and the specific science products listed in Table 3 will be published.

The four science programs are led by Tommaso Treu (UCLA) for dark matter/energy; Andrea Ghez, Tuan Do and Mark Morris (UCLA) for the Galactic Center; Shelley Wright (UCSD), Tucker Jones (UCD) and Claire Max (UCSC) for galaxy evolution; and Michael Liu (UH) and Dimitri Mawet (Caltech) for gas-giant protoplanets. In addition, Jessica $\mathrm{Lu}(\mathrm{UCB})$ is the KAPA project scientist. 
Table 2: KAPA science data available through the Keck Observatory Archive.

\begin{tabular}{|c|c|c|c|c|c|}
\hline Science Case & Reduced Data Sets in KOA & \# targets & \# nights & OSIRIS & Band \\
\hline \multirow{2}{*}{ Dark Matter } & Gravitational imaging & 20 & 5 & Imager & \multirow{4}{*}{$\mathrm{H}$ or $\mathrm{K}$} \\
\hline & Lensed quasar flux ratios & 20 & 10 & IFU & \\
\hline \multirow{2}{*}{ Dark Energy } & Lensed host galaxy images & 40 & 10 & Imager & \\
\hline & Stellar kinematics of stars in the deflector & 40 & 17 & IFU & \\
\hline \multirow{2}{*}{$\begin{array}{l}\text { Galactic } \\
\text { Center }\end{array}$} & Astrometric imaging (central 1" diameter) & 100 & 12 & Imager & $\mathrm{H}, \mathrm{K}$ \\
\hline & Radial velocity spectroscopy (central 1" dia.) & 25 & 28 & IFU & $\mathrm{H}, \mathrm{K}$ \\
\hline $\begin{array}{c}\text { Galaxy } \\
\text { Evolution }\end{array}$ & Gravitationally-lensed galaxies & 40 & 20 & IFU & $\mathrm{J}, \mathrm{H}, \mathrm{K}$ \\
\hline \multirow{2}{*}{$\begin{array}{c}\text { Gas-Giant } \\
\text { Protoplanets }\end{array}$} & First-epoch imaging & 200 & 30 & Imager & $\mathrm{K}$ \\
\hline & Astrometric \& spectroscopic followup & TBD & $\geq 5$ & IFU & K \\
\hline
\end{tabular}

Table 3: KAPA science data products.

\begin{tabular}{|c|l|}
\hline Science Case & \multicolumn{1}{|c|}{ Publicly Available Data Products } \\
\hline Dark Matter & Subhalo mass function \\
\hline Dark Energy & Stellar velocity fields \\
\hline \multirow{2}{*}{ Galactic } & Kinematics catalog \\
\cline { 2 - 2 } Center & Reference frame \\
\cline { 2 - 2 } & Stellar spectral \& photometric properties \\
\hline Galaxy & Internal kinematics of lensed galaxies \\
\cline { 2 - 2 } Evolution & Metallicity gradients of lensed galaxies \\
\hline \multirow{2}{*}{$\begin{array}{c}\text { Gas-Giant } \\
\text { Protoplanets }\end{array}$} & Contrast curves \\
\cline { 2 - 2 } & List of identified candidates \\
\cline { 2 - 2 } & Imaging data \\
\hline
\end{tabular}

\section{EDUCATION PROGRAM}

The overall goal of the KAPA education program is to broaden participation in astronomical instrumentation to include more women and underrepresented minorities. This program (see Figure 21) includes hosting scholars through the Akamai and Keck Visiting Scholars (KVS) programs, developing a new one-week instrumentation summer school called AstroTech funded by the Heising-Simons Foundation and led by Lisa Hunter and Jessica Lu, and employing about eight KAPA science postdocs and a KAPA technology postdoc.

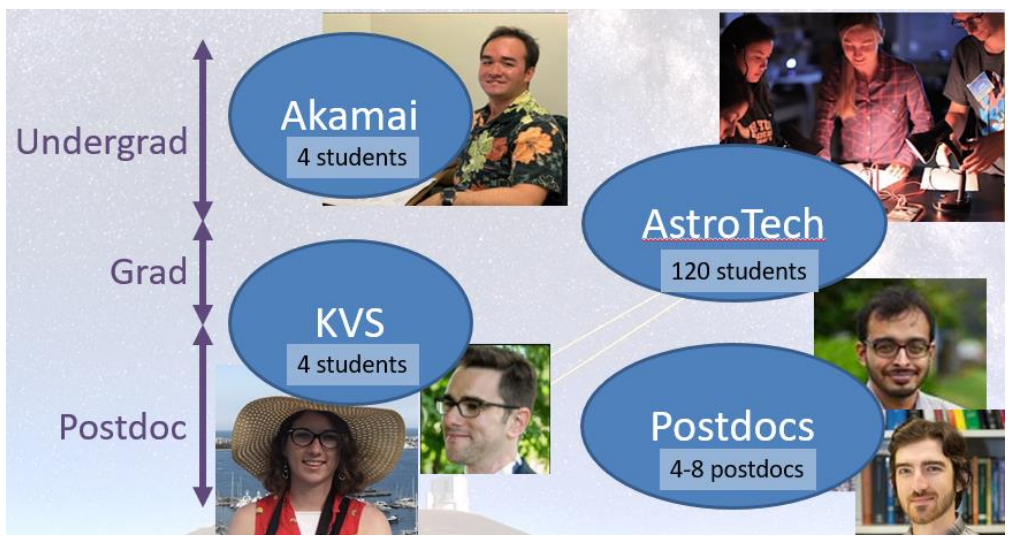

Figure 21: KAPA education program designed to broaden participation in instrumentation for women and underrepresented minorities.

During the summer of 2019, the first Akamai student participated in the development of the new laser safety system, the technology postdoc began work at Keck, and an AstroTech development workshop was held to prototype and design summer school activities. Unfortunately the summer 2020 Akamai and AstroTech programs were cancelled 
due to COVID-19. Keck Visiting Scholars joined us in October 2019 and August 2020 to work on PSF-R and prototype operations software sequencers, respectively.

\section{STATUS AND PLANS}

Table 4 provides a view of the project's technical status from the perspective of completed and planned reviews. In additional annual KAPA science team workshops are held each September to discuss and develop the science tools and programs.

Table 4: KAPA reviews completed (left) and planned (right). The design reviews include system, preliminary and detailed (SDR, PDR, DDR) and are followed by a pre-install review and an operations handover.

\begin{tabular}{|c|l|}
\hline Date & \multicolumn{1}{|c|}{ Completed Reviews } \\
\hline $9 / 26 / 18$ & WMKO Real-time Controller (RTC) PDR \\
\hline $12 / 13 / 18$ & Microgate RTC PDR \\
\hline $1 / 31 / 19$ & Laser Infrastructure Design I \\
\hline $5 / 2 / 19$ & Laser Infrastructure Design II \\
\hline $7 / 24 / 19$ & Microgate RTC DDR \\
\hline $9 / 12 / 19$ & Laser Tomography (LT) SDR \\
\hline $9 / 12 / 19$ & WFS Reducer Optics DDR \\
\hline $2 / 13 / 20$ & WMKO RTC DDR \\
\hline $5 / 7 / 20$ & Laser Operations (Ops) Handover \\
\hline $6 / 3 / 20$ & OMP Asterism Generator (AG) PDR \\
\hline $6 / 17 / 20$ & WMKO AG PDR \\
\hline $11 / 19 / 20$ & LT PDR \\
\hline $11 / 20 / 20$ & WFS Pupil Relay DDR \\
\hline $11 / 20 / 20$ & Asterism Simulator (AS) DDR \\
\hline
\end{tabular}

\begin{tabular}{|c|l|}
\hline Date & \multicolumn{1}{|c|}{ Planned Reviews } \\
\hline $12 / 15 / 20$ & OMP AG DDR \\
\hline $1 / 10 / 21$ & RTC Factory Acceptance \\
\hline $1 / 18 / 21$ & WMKO AG DDR \\
\hline $1 / 31 / 21$ & Keck I RTC + OCAM Pre-install \\
\hline $2 / 15 / 21$ & AS Pre-install \\
\hline $3 / 30 / 21$ & WFS Pupil Relay Pre-install \\
\hline $6 / 3 / 21$ & LT DDR \\
\hline $6 / 15 / 21$ & Keck II RTC + OCAM Pre-install \\
\hline $9 / 28 / 21$ & AG Acceptance \\
\hline $9 / 30 / 21$ & Keck I RTC + OCAM Ops Handover \\
\hline $10 / 12 / 21$ & AG Pre-install \\
\hline $12 / 30 / 21$ & Keck II RTC + OCAM Ops Handover \\
\hline $1 / 30 / 23$ & Laser Tomography Ops Handover \\
\hline
\end{tabular}

\section{CONCLUSION}

The five year KAPA program will upgrade the Keck I AO system with a new higher return sodium-wavelength laser, a new real-time controller for higher bandwidth and increased capacity to support both KAPA and future upgrades, multiple LGS for laser tomography, multiple near-infrared tip-tilt sensing, and PSF-R for the KAPA science observations with OSIRIS. The AO correction and sky coverage are predicted to be significantly improved as a result. The resultant KAPA system will be used to carry out four key science programs as well as supporting all science observations with the Keck I AO system. A number of science tools are being developed to support observation planning and data analysis. An extensive education program is in place, and has and will be integrated with KAPA technical developments where possible.

\section{ACKNOWLEDGEMENTS}

The W. M. Keck Observatory is operated as a scientific partnership among the California Institute of Technology, the University of California, and the National Aeronautics and Space Administration. The Observatory was made possible by the generous financial support of the W. M. Keck Foundation. Funding support for the KAPA system provided by the National Science Foundation Mid Scale Innovations Program award AST-1836016 (PI: Wizinowich). The Keck II real-time controller is funded by the NSF Major Research for Instrumentation Program award AST-1727071 (Wizinowich). Funding support for the KAPA science programs is provided by the Gordon and Betty Moore Foundation through awards to UH (Liu), UCSD (Wright) and two awards to UCLA (Ghez and Treu), and sub-awards to CIT (Mawet), UCB (Lu) and UCD (Jones). Funding support for the AstroTech instrumentation intensive is provided by the Heising-Simons Foundation through UCSC (Hunter) and UCB $(\mathrm{Lu})$. The turbulence simulator is supported by a grant from R. Simperman.

\section{REFERENCES}

[1] Chin, J., et al., “Keck I Laser Guide Star Adaptive Optics System,” Proc. SPIE 8447 (2012). 
[2] Larkin, J., et al., "OSIRIS: A diffraction limited integral field spectrograph for Keck," New Astronomy Reviews 50 (2006).

[3] Chin, J., et al., "Keck II laser guide star adaptive optics system and performance with the TOPTICA/MPBC laser," Proc. SPIE 9909 (2016).

[4] Correia, C. et al., "Static and predictive tomographic reconstruction for wide-field multi-object adaptive optics systems," JOSA A 31, 101 (2014).

[5] Correia, C. et al., "Spatio-angular minimum-variance tomographic controller for multi-object adaptive-optics systems," Applied Optics 54, 5281 (2015).

[6] Femenia-Castella, B., et al., "Status and new developments with the Keck I near-infrared tip-tilt sensor," Proc. SPIE 9909 (2016).

[7] Plantet, C., et al., "LIFT on Keck: analysis of performance and experiments towards on-sky validation,” AO4ELT5 (2017).

[8] Ragland, S., et al., "Status of Point Spread Function Determination for Keck Adaptive Optics," Proc. SPIE 10703 (2018).

[9] Beltramo-Martin, O., et al., "Parametric point spread function for single-conjugated adaptive optics assisted observations," MNRAS in-press (2019).

[10] Do, T., et al., "Point-spread function reconstruction for integral-field spectrograph data," Proc. SPIE 10703 (2018).

[11] Ciurlo, A., et al., "Off-axis PSF reconstruction for integral field spectrograph: instrumental aberrations and application to Keck/OSIRIS data,” Proc. SPIE 10703 (2018).

[12] Beltramo-Martin, O. et al. PRIME: Psf Reconstruction and Identification for Multiple sources characterization Enhancement. MNRAS 487, 5450 (2019).

[13] Jia, S., et al. "The Galactic Center: Improved Relative Astrometry for Velocities, Accelerations and Orbits near the Supermassive Black Hole," ApJ 873, 9J (2019).

[14] Do, T., et al., "Relativistic redshift of the star S0-2 orbiting the Galactic Center supermassive black hole," Science 365, 644D (2019). 